\title{
Estradiol-Induced Inhibition of Body Weight Gain and Reduction of Food Intake Are Not Associated with Diurnal Rhythmicity
}

\author{
Hiroshi MIURA, Takefumi KIKUSUI, Yukari TAKEUCHI and Yuji MORI \\ Laboratory of Veterinary Ethology, The University of Tokyo, Yayoi 1-1-1, \\ Bunkyo-ku, Tokyo 113-8657, Japan
}

\begin{abstract}
Ovariectomized female rats were subcutaneously implanted with silicone capsules containing estradiol $(n=11)$, which maintained physiological levels of plasma estradiol, or cholesterol $(n=10)$ as control at the age of 9 weeks. Body weight, amount of food intake and water consumption were recorded twice daily during a 2 week period starting one week before the capsule implantation. Estradiol treatment induced suppression of daily food intake and body weight gain in animals carrying estradiol capsules as compared with those carrying cholesterol one, whereas there was no apparent estradiol effect on water intake. The extent of suppression of both body weight gain and food intake were indistinguishable between the light and the dark phases. The present results suggest that the mechanism which mediates the inhibition of body weight gain and the reduction of food intake is not directly linked with that generating diurnal rhythmicity.

Key words: Estradiol, Body weight, Food intake, Diurnal rhythm.
\end{abstract}

(J. Reprod. Dev. 45: 259-264, 1999)

$\mathbf{E}_{\mathrm{i}}^{\mathrm{s}}$ stradiol secreted by ovarian follicles possesses inhibitory effects on feeding behavior as well as somatic growth, as the female rat feeds more and gains body weight following ovariectomy and these changes can be nullified by exogenously administering estradiol [1-3]. The physiological meaning of this estradiol-induced growth retardation is postulated to be that increased estradiol secretion from puberty onward limits the somatic growth at a certain level of body size which is sufficient for performing reproduction so that a female can re-direct available energy toward successful reproductive accomplishment rather than its own growth in an environment with limited resources.

With this experimental model it has often been interpreted that the growth retardation is simply the result of decreased food consumption, but this

Accepted for publication: April 9, 1999

Correspondence: Y. Mori might not always be the case. Wade \& Schneider [4] have argued that there is no direct causative relationship between these two parameters based on the results of their own and other researchers, which include the finding that estradiol-induced reduction of food intake was not necessarily accompanied by suppression of body weight gain, or vice versa. However, since the body weight increases after feeding, it has been difficult to assess accurately whether or not there are actual interactions between the two parameters.

There are apparent diurnal differences of the estradiol effect on some physiological parameters such as locomotor activity [5-7], and so it appears plausible that estradiol affects the diurnal rhythmicity of body weight and food intake as well. In this study, to testify this possibility we measured the day/night changes of three metabolic parameters, namely, the body weight, the amounts of food intake and the water consumption, and compared them in two groups of ovariectomized rats which 
were treated with either estradiol or cholesterol.

\section{Materials and Methods}

\section{Animals}

Female Wistar rats $(\mathrm{n}=21)$ were ovariectomized at the age of 7 weeks to eliminate the influence of gonadal hormones, and divided into two groups according to the treatment they received (estradiol or control) as described below. They were individually caged in a climate controlled room, where the temperature and relative humidity were maintained at $25 \pm 2 \mathrm{C}$ and $70 \pm 5 \%$, respectively, under 12L12D (light phase between 8:00 and 20:00). They were allowed free access to food (Rat MR breeder, Nippon Nousan) and water.

\section{Subcutaneous capsules for estradiol treatment}

Silicone tubes ( $2 \mathrm{~mm}$ i.d., $3 \mathrm{~mm}$ o.d. and $7.5 \mathrm{~mm}$ in length) were filled with a mixture of estradiol and cholesterol powder $(1: 4, \mathrm{w} / \mathrm{w})$ and sealed both ends with silicone glue. This size of estradiol capsule was reported to maintain physiologically high levels of blood estradiol (about $50 \mathrm{pg} / \mathrm{ml}$ ), equivalent to peak concentrations at proestrus in intact female rats [8]. All the capsules were soaked in sterilized physiological saline solution overnight prior to the implantation.

\section{Measurement of body weight, amount of food and water intake}

After a one week recovery period from the ovariectomy, the three parameters, namely body weight (BW), food intake (FI) and water intake (WI) were recorded twice daily (immediately after the light on and before the light off) for two weeks. In the middle of this two weeks period, the animals were divided into two groups so that there were no differences in any of the three parameters between the two groups. Eleven ovariectomized rats were implanted subcutaneously, under ether anesthesia, with one estradiol capsule, and the remaining 10 animals were implanted with cholesterol capsules as control.

\section{Data analysis}

The BW data were standardized to the value of the implantation day. The mean values for BW change, FI and WI during the light phase and the dark phase were recorded and averaged for each of the 7 day periods, i.e. before and after the im-

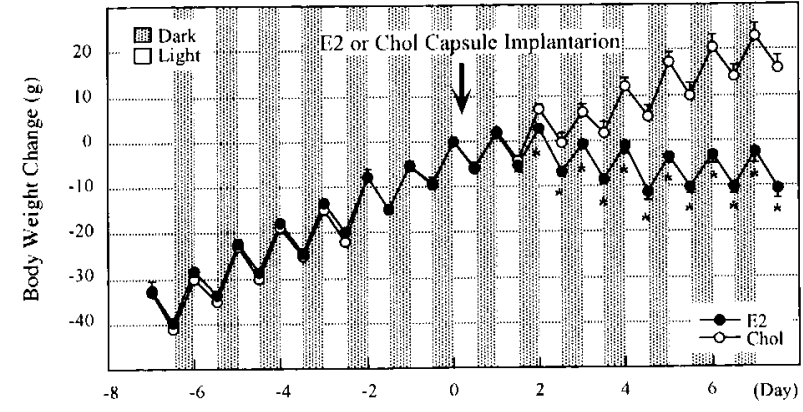

Fig. 1. Changes in the $B W$ in ovariectomized rats which were subcutaneously implanted with capusules containing estradiol (E2, $\mathrm{n}=11$ ) or cholesterol (Chol, $\mathrm{n}=10$ ) at Day 0. Data are means \pm SEM. There was a significant difference in the trends between the two groups (ANOVA, $\mathrm{p}<0.05$ ).

${ }^{*} \mathrm{P}<0.01$ as compared with the Chol group (Student's $t$-test).

plantation of capsules. The data were expressed as means \pm SEM, and the differences between the groups were assessed by ANOVA or Student's $t$ test.

\section{Results}

Sequential changes of BW in ovariectomized rats implanted subcutaneously with silicone capsules containing either estradiol or cholesterol on Day 0 are shown in Fig. 1. There was a gradual increase of BW with apparent diurnal fluctuations in which BW increased during the dark phase and decreased during the light phase. Although the baseline continued to rise similarly in both groups until the day of estradiol implantation, the gradual increase of BW ceased following subcutaneous implantation of the estradiol capsule (but not the cholesterol), and the rats bearing estradiol capsules eventually became lighter $(p<0.05)$ than those carrying cholesterol capsules. However, the pattern of diurnal fluctuation of BW was unchanged in both groups.

The data on BW change were further analyzed by measuring three components, namely, the light phase decrease, the dark phase increase, and the total balance within a given day. These values were averaged for the pre- and post-implantation one week periods to determine the significant effecst of the estradiol treatment. The method for measuring these components are schematically described in Fig. 2. Likewise, the other two 


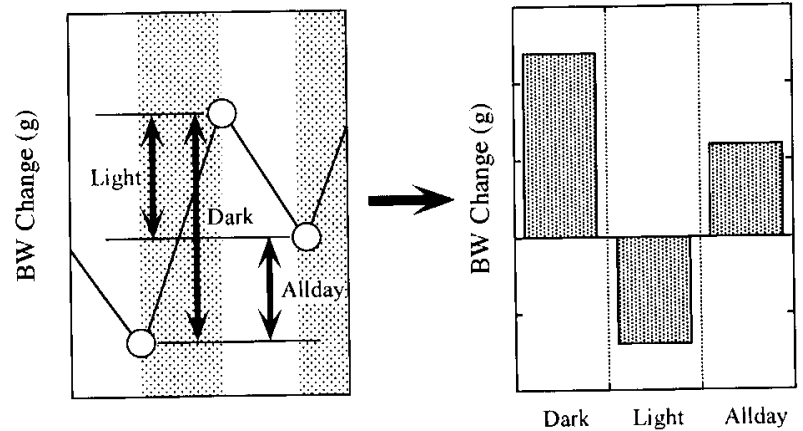

Fig. 2. Schematic illustration of the method for measuring two components of BW change; the dark phase increase, the light phase decrease and the balance within a day.

parameters, namely, amounts of FI and WI were mathematically processed.

The effects of estradiol treatment on the daily BW fluctuation, FI, and WI are summarized in Fig. 3. There was no difference between the two groups during the pre-implantation control week. The data of the post-implantation week were virtually identical to those of the pre-implantation week in the control group. In contrast, in the estradiol treated group, the light-phase BW decrease was enhanced. The amount of FI was also suppressed by estradiol treatment throughout one day. On the other hand, the WI was not affected by estradiol treatment.

The effects of estradiol on these parameters were highlighted by comparing the mean values for the pre- and post-implantation weeks in each group (Fig. 4 left), and then by calculating the differences between the two groups which were considered to represent the component directly related to estradiol treatment (Fig. 4 right). The extent of estradiol-induced suppression was similar between the dark and light phases for both BW and FI, and total daily inhibition by estradiol of BW gain and of FI were $3.81 \mathrm{~g}$ and $3.42 \mathrm{~g}$, respectively.

Based on these observations, schematical illustrations were drawn to characterize the relationship between estradiol-induced suppression and the diurnal fluctuation of BW and FI (Fig. 5). Chronic administration of estradiol affected the baseline increase of BW without influencing the diurnal pattern of its fluctuation at all. Similarly, the difference between dark- and light-phases was also maintained for the FI, although the average value decreased slightly.

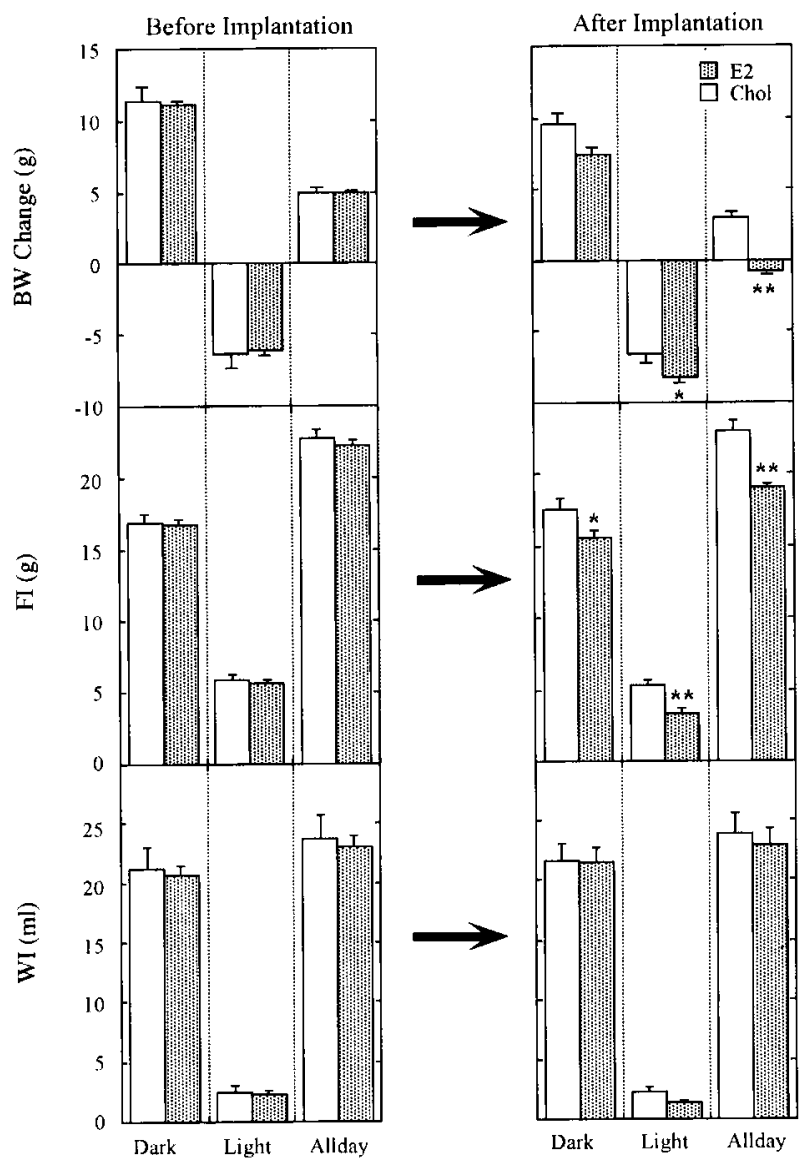

Fig. 3. Mean values for daily BW change, FI and WI at the weeks before and after estradiol (E2) or cholesterol (Chol) implantation in the dark phase, the light phase and all day. Values are the mean \pm SEM for 10-11 animals. * $\mathrm{P}<0.05,{ }^{* *} \mathrm{P}<0.01$ between the two groups (Student's $t$ test).

\section{Discussion}

The continuous administration of estradiol via the subcutaneously implanted capsule resulted in the suppression of the amount of FI and BW gain in young ovariectomized female rats. The result is summarized in Fig. 6. This effect of estradiol on the $\mathrm{BW}$ is considered to be ascribed, at least in part, to the suppression of body fat accumulation [9-14]. The results of the present study clearly show that these inhibitory effects of estradiol on the FI and the BW gain are exerted throughout the day without any appreciable difference in the extent of suppression between the light and dark phases. Diurnal rhythmicity of feeding behavior is under the control of the suprachiasmatic nucle- 


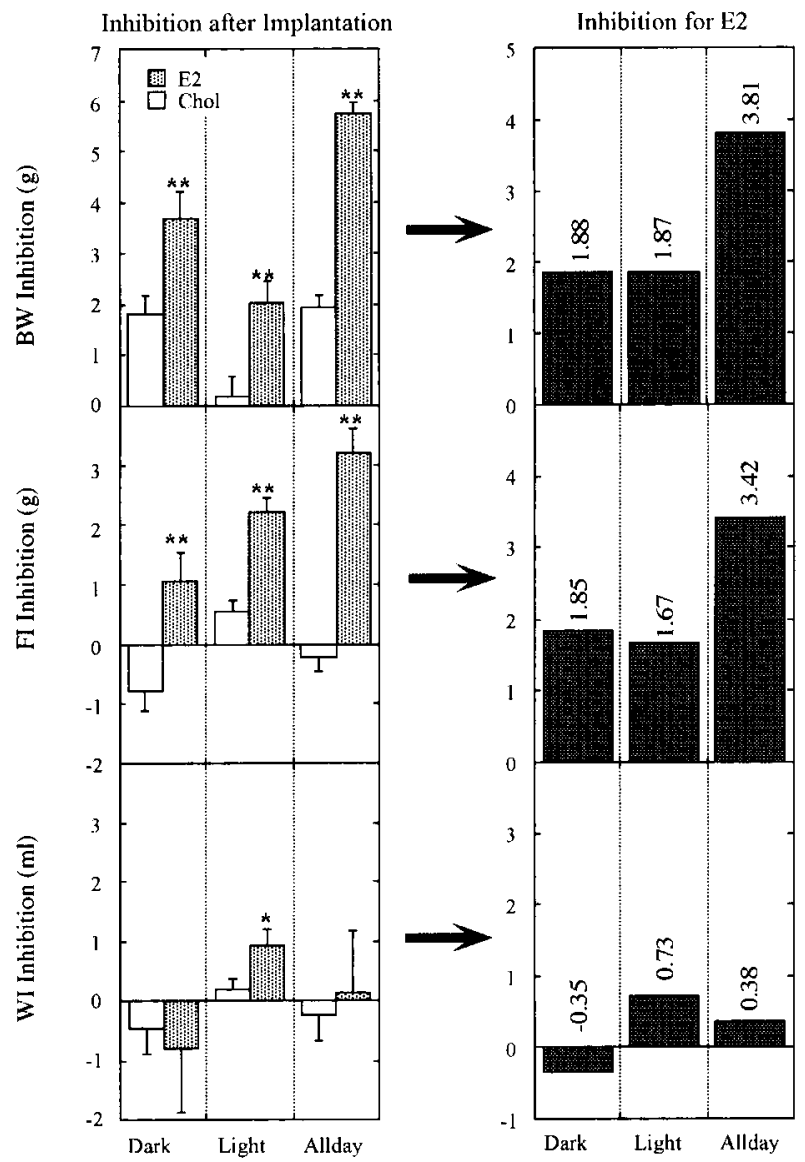

Fig. 4. Amounts of inhibition of BW gain (top), FI (middle) and WI (bottom) during estradiol (E2) or cholesterol (Chol) treatments. The estradiol-induced changes are detailed on the right. Values are means \pm SEM for 10-11 animals. ${ }^{*} \mathrm{P}<0.05,{ }^{* *} \mathrm{P}<0.01$ as compared with the control Chol group (Student's $t$ test).

us of the hypothalamus as is the case for other biological rhythms such as body temperature, activity and the immune function $[15,16]$. Therefore, the observation that estradiol suppressed equally both the daytime and nighttime components of FI suggests that the mechanism conveying the estradiol effect may not be directly linked to the circadian oscillator. In other words, estradiol may not influence the biological clock function, but it may alter the metabolic set-points, which are manifested by physiological parameters such as the amount of FI and the body-fat content.

Previous studies have suggested the involvement of two hypothalamic nuclei, namely, the paraventricular nucleus (PVN) and the ventromedial hypothalamus $(\mathrm{VMH})$ in the control of feeding be-

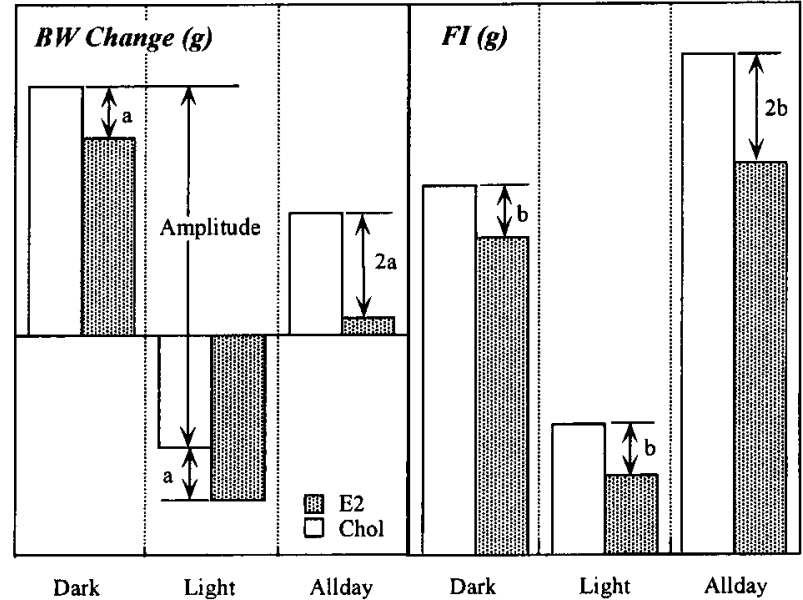

Fig. 5. Schematic illustration of the estradiol effects on the BW and FI. The inhibition of both BW and FI are the same between dark and light phases. The extent of inhibition of BW gain by E2 treatment is shown as "a" (left panel) and that of FI as "b" (right panel).
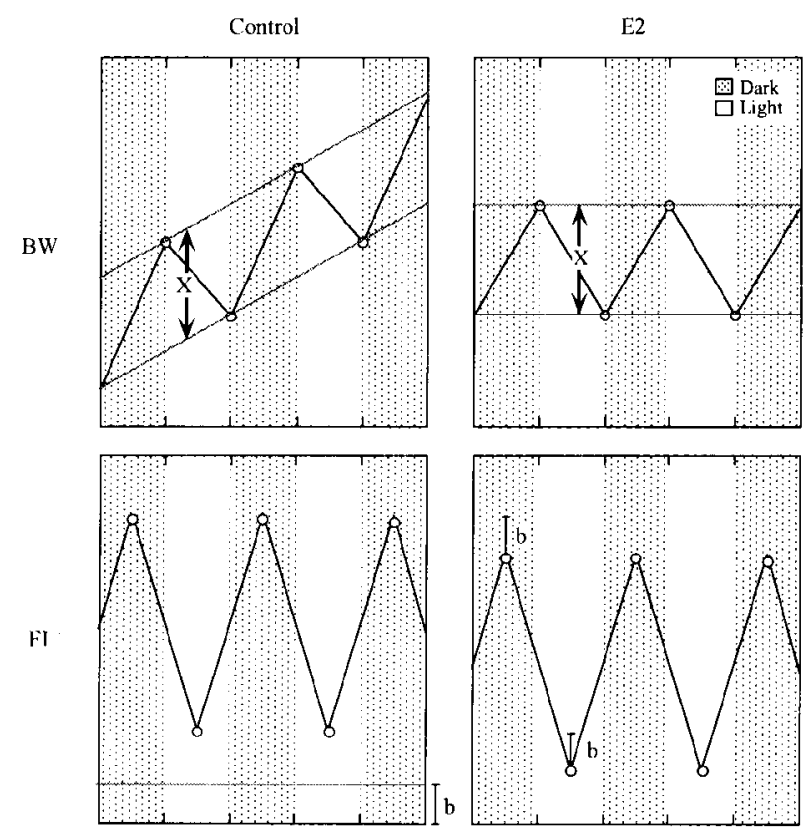

Fig. 6. The schematic illustration of how estradiol (E2) influence BW and FI. E2 suppresses the gradual increase of BW but does not influence the amplitude of daily fluctuation. E2 also reduces FI, and the extent of suppression is similar between dark and light phases. Therefore the effect of E2 appears independent of diurnal rhythmicity.

havior. The function of these nuclei, however, may differ from each other, since the local administration of estradiol into the PVN but not to the VMH 
suppressed FI [17]. Lesioning of these nuclei, on the other hand, increased the amount of FI, but again the pattern of excessive eating was different between the PVN and VMH; there was no diurnal rhythmicity of feeding behavior in the rat with VMH lesion whereas the rhythm remained in the PVN-lesioned animal [18]. Based on the results of these studies, the VMH is considered to be directly involved in the expression of the diurnal pattern of feeding behavior. In contrast, the involvement of the PVN in the circadian rhythmicity appears to be rather indirect, and so it can be speculated that some of the central action of estradiol is mediated via the neural network which includes the PVN. It appears that the present results support this view.

The mechanisms involved in the BW change may be more complicated than those involved in the alteration of feeding behavior as it is the sum of various metabolic processes. In the present study, it was possible to divide BW change into two components. One is the basal component of which steady growth was influenced by the estradiol treatment, and the other is the diurnal fluctuation component which was not affected by estradiol. Rats are nocturnal species, and so the majority of feeding episodes occur during the dark phase. Although we did not measure the elimination in this study, the effect of estradiol on elimination, if any, could also be independent of the circadian system, because no difference was seen in the actual loss of body weight gain.

The averaged daily loss of BW gain induced by estradiol $(3.81 \mathrm{~g})$ was fairly close to the averaged daily suppression of FI (3.42 g). Despite the similarity of these two figures, it would be still premature to postulate the causative relationship between the two parameters, since we do not know yet how much energy is being expended for the self-maintaining activities such as thermoregulation and locomotion. In relation to this notion, Butera et al. [19] reported that suppressed feeding was not accompanied by body weight loss in their experiment with PVN lesion, and Wade \& Schneider [4] mentioned some cases of discrepancies between the two parameters. Therefore, the alternative explanation may be that two relatively independent mechanisms are involved in the mediation of estradiol effects on the feeding behavior and the BW gain, which appears more plausible than hypothesizing a causative relationship between the two. Further investigation is needed to clarify whether or not this is actually the case.

In the present study, we investigated the pattern of estradiol-induced inhibition of FI and of BW gain with special reference to the fluctuation between the light and dark phases. We have concluded that estradiol exerts these inhibitory effects via the mechanisms which may not be directly linked to the neural components responsible for the generation of diurnal rhythmicity.

\section{Acknowledgements}

This study was partly supported by grants-inaid for scientific research from Ministry of Education, Science, Sports and Culture, and Research for the Future Program from the Japan Society for the Promotion of Science (JSPS-RFTF 97L00904).

\section{References}

1. Gentry RT, Wade GN, Roy EJ. Individual differences in estradiol-induced behaviors and neural ${ }^{3} \mathrm{H}$-estradiol uptake in rats. Physiol Behav 1976; 17: 195-200.

2. Sieck GC, Nance DM, Gorski RA. Estrogen modification of feeding behavior in the female rat: Influence of metabolic state. Physiol Behav 1978; 21: 893-897.

3. Tarttelin MF, Gorski RA. The effects of ovarian steroids on food and water intake and body weight in the female rat. Acta Endocrinologica 1973; 72: 551-568.

4. Wade GN, Schneider JE. Metabolic fuels and re- production in female mammals. Neuroscience and Biobehavioral reviews 1992; 16: 1-38.

5. Gerall AA, Napoli AM, Cooper UC. Daily and hourly estrous running in intact, spayed, and estrone implanted rats. Physiol Behav 1973; 10: 225-229.

6. Kennedy GC. Hypothalamic control of the endocrine and behavioral changes associated with oestrus in the rat. J Physiol 1964; 172: 383-392.

7. Young WC, Fish WR. The ovarian hormones and spontaneous running activity in the female rat. Endocrinology 1945; 36: 181-189.

8. Albert DJ, Jonik RH, Gorzalka BB, Newlove T, 
Webb B, Walsh ML. Serum estradiol concentration required to maintain body weight, attractivity, proceptivity, and receptivity in the ovariectomized female rat. Physiol Behav 1991; 49: 225-231.

9. Benoit V, Vallette B, Mercier L, Meignen JM, Boyer J. Potentiation of epinephrine-induced lipolysis in fat cells from estrogen treated rats. Biochem Biophys Res Comm 1982; 109: 1186-1191.

10. Hansen L, Fahmy N, Nielsen JH. The influence of sexual hormones on lipogenesis and lipolysis in rat fat cells. Acta Endocrinol 1980; 95: 566-570.

11. Lacasa D, Agli B, Pecquery R, Giudicelli Y. Influence of ovariectomy and regional fat distribution on the membranous transducing system controlling lipolysis in rat fat cells. Endocrinology 1991; 128: 747-753.

12. Lazzarini SJ, Wade GN. Role of sympathetic nerves in effects of estradiol on rat white adipose tissue. Am J Physiol 1991; 260: R47-51.

13. Pasquier YN, Pecquery R, Giudicelli Y. Increased adenylate cyclase catalytic activity explains how estrogens "in vivo" promote lipolytic activitiy in rat white fat cells. Biochem Biuophys Res Comm 1988; 154: 1151-1159.
14. Rebuffé-Scrive M. Sex steroid hormones and adipose tissue metabolism in ovariectomized and adrenalectomized rats. Acta Physiol Scand 1987; 129: 471-477.

15. Nagai K, Nishio T, Nakagawa H, Nakamura S, Fukuda Y. Effect of bilateral lesions of the suprachiasmatic nuclei on the circadian rhythm of food-intake. Brain Research 1978; 142: 384-389.

16. Stoynev AG, Ikonomov OC. Feeding pattern and light-dark variations in water intake and renal excretion after suprachiasmatic nuclei lesions in rats. Physiol Behav 1982; 29: 35-40.

17. Butera PC, Beikirch RJ. Central implants of diluted estradiol: independent effects on ingestive and reproductive behaviors of ovariectomized rats. Brain Research 1989; 491: 266-273.

18. Tokunaga K, Fukushima M, Kemnitz JW, Bray GA. Comparison of ventromedial and paraventricular lesions in rats that become obese. Am J Physiol 1986; 251: R1221-1227.

19. Butera PC, Willard DM, Raymond SA. Effects of PVN lesions on the responsiveness of female rats to estradiol. Brain Res 1992; 576: 304-310. 